\title{
Efficacy and Safety of Pitavastatin in Children and Adolescents with Familial Hypercholesterolemia in Japan and Europe
}

\author{
Mariko Harada-Shiba ${ }^{1}$, John J.P. Kastelein², G. Kees Hovingh ${ }^{2}$, Kausik K. Ray ${ }^{3}$, Akira Ohtake ${ }^{4}$, \\ Osamu Arisaka ${ }^{5}$, Takao Ohta ${ }^{6}$, Tomoo Okada ${ }^{7}$, Hideki Suganami ${ }^{8}$ and Albert Wiegman ${ }^{9}$ \\ ${ }^{1}$ Department of Molecular Innovation in Lipidology, National Cerebral and Cardiovascular Center Research Institute, Osaka, Japan \\ ${ }^{2}$ Department of Vascular Medicine, Academic Medical Center, University of Amsterdam, the Netherlands \\ ${ }^{3}$ Department of Primary Care and Public Health, School of Public Health, Imperial College London, London, UK \\ ${ }^{4}$ Department of Pediatrics, Faculty of Medicine, Saitama Medical University, Saitama, Japan \\ ${ }^{5}$ Department of Pediatrics, Dokkyo Medical University School of Medicine, Tochigi, Japan \\ ${ }^{6}$ Department of Child Health and Welfare (Pediatrics), Faculty of Medicine, University of the Ryukyu, Nishihara, Japan \\ ${ }^{7}$ Department of Pediatrics and Child Health, Nihon University School of Medicine, Tokyo, Japan \\ ${ }^{8}$ Clinical Data Science Department, Kowa Company, Ltd., Tokyo, Japan \\ ${ }^{9}$ Department of Pediatrics, Academic Medical Center, University of Amsterdam, the Netherlands
}

\begin{abstract}
Aim: Children with Familial Hypercholesterolemia (FH) are widely prescribed statins, and it has been suggested that the effects of statins differ among ethnicities. We compared the efficacy and safety of pitavastatin in children and adolescents with $\mathrm{FH}$ in clinical trials conducted in Japan and Europe.

Methods: Low-density lipoprotein cholesterol (LDL-C) reductions, adjusted for confounding factors, and safety were compared between the studies in Japan and Europe. In the Japanese study, 14 males with heterozygous $\mathrm{FH}$, aged $11.8 \pm 1.6$ years, were randomized to 52 -week double-blind treatment with 1 or $2 \mathrm{mg} /$ day pitavastatin. In the European study, 106 children and adolescents with high risk hyperlipidemia (103 heterozygous $\mathrm{FH}$ ), aged 10.6 \pm 2.9 years, were randomized to 12 -week doubleblind treatment with 1,2 or $4 \mathrm{mg} /$ day pitavastatin or placebo; 84 of these patients and 29 new patients participated in a 52-week open-label extension study.

Results: Age, body weight and baseline LDL-C were identified as factors influencing LDL-C reduction. There were no significant differences in the adjusted mean percentage reduction in LDL-C in Japanese and European children by pitavastatin $(24.5 \%$ and $23.6 \%$, respectively at $1 \mathrm{mg} / \mathrm{day}$ and $33.5 \%$ and $30.8 \%$, respectively at $2 \mathrm{mg} /$ day). Pitavastatin was well tolerated without any difference in the frequency or nature of adverse events between the treatment groups, or between the studies.

Conclusion: There were no significant differences between the efficacy or safety of pitavastatin in Japanese and European children and adolescents with FH, suggesting no relevant ethnic differences in the safety or efficacy of pitavastatin.
\end{abstract}

Key words: Familial hypercholesterolemia, Children, Pitavastatin, Low-density lipoprotein cholesterol, Ethnic difference

\section{Introduction}

Familial hypercholesterolemia $(\mathrm{FH})$ is an autoso-

Address for correspondence: Mariko Harada-Shiba, Department of Molecular Innovation in Lipidology, National Cerebral and Cardiovascular Center Research Institute, 5-7-1 Fujishiro-dai, Suita, Osaka 565-8565, Japan

E-mail: mshiba@ncvc.go.jp

Received: August 4, 2017

Accepted for publication: October 7, 2017 mal dominant disorder that results in defective lowdensity lipoprotein (LDL) receptor function. The prevalence of $\mathrm{FH}$ has been estimated as approximately 1 in 500 people for heterozygotes and 1 in a million for homozygotes, but recently higher prevalences were reported ( 1 in 200 people for heterozygotes and 1 in 200,000 to 300,000 for homozygotes) although there is a significant variation across the world ${ }^{1-4)}$. This would imply that approximately 0.6 and 4.5 million $\mathrm{FH}$ patients are present in Japan and Europe, respectively. The objec- 
tive in the management of $\mathrm{FH}$ is to temper the progression of atherosclerosis by reducing LDL-cholesterol (LDL-C) levels and early diagnosis and therapeutic intervention are therefore pivotal ${ }^{5,6}$. In Western countries where the risk of coronary artery disease is higher than in Japan ${ }^{7,8)}$, guidelines for the treatment of FH recommend the early initiation of a cholesterollowering therapy even for children and recommend 3-hydroxy-3-methylglutaryl-coenzyme A (HMG$\mathrm{CoA}$ ) reductase inhibitors (statins) as first-line therapy for children with heterozygous $\mathrm{FH}^{2,6,9,10)}$. The guidelines for treatment of pediatric $\mathrm{FH}$ in Japan have recently been updated to change the recommended first-line therapy from bile acid sequestrants to statins ${ }^{11)}$.

Pitavastatin was first approved in Japan in 2003 for the treatment of hypercholesterolemia and $\mathrm{FH}$ in adults and, to date, has been approved in 43 countries including in Europe and the US. Clinical studies in Japan and Europe in children and adolescents with FH confirmed the significant LDL-C lowering effect of pitavastatin and also showed this statin to be well tolerated ${ }^{12,13)}$. These findings led to the approval in Japan of pitavastatin for treatment of children and adolescents with FH in June 2015, thereby being the first statin in Japan approved for this indication. In Europe, the data for those clinical studies will shortly be submitted to regulators for inclusion in the Summary of Product Characteristics (SmPC).

As reported in the original paper of the study in Japan $^{12)}$, pitavastatin resulted in potent LDL-C reduction in children and adolescents with $\mathrm{FH}$ aged 10-15 years, with $27.3 \%$ and $34.3 \%$ reduction in LDL-C at doses of 1 and $2 \mathrm{mg}$ daily, respectively. Similarly, in trials conducted in Europe ${ }^{13)}$, pitavastatin was shown to lower LDL-C levels by $23.5 \%, 30.1 \%$, and $39.3 \%$ at doses of 1,2 , and $4 \mathrm{mg}$ daily, respectively in children and adolescents with FH aged 6-17 years. The LDL-C lowering efficacy of pitavastatin in the study in Japan was numerically higher than that in Europe. However, lifestyle, healthcare access and disease management differ between the regions. The studies also differed in terms of the inclusion criteria including age, gender, and baseline LDL-C level, primary endpoints, and analytical methods, since the settings for each study were separately decided based on discussion with the regulatory authorities in Japan and Europe. Therefore, exploratory analyses were carried out to identify factors affecting the LDL-C lowering efficacy of pitavastatin. The LDL-C reductions in the studies were recalculated using the same methodology and adjusted for identified confounding factors to allow comparison of the efficacy and safety of pitavastatin in Japanese and European children and adolescents with $\mathrm{FH}$.

\section{Aim}

To compare the efficacy and safety of pitavastatin in Japanese and European children and adolescents with $\mathrm{FH}$.

\section{Methods}

\section{Japanese Study ${ }^{12)}$}

A randomized, double-blind, parallel study was conducted at 16 facilities in Japan to evaluate the efficacy and safety of pitavastatin in Japanese boys with clinically diagnosed heterozygous $\mathrm{FH}$ aged $10-15$ years. Patients with homozygous FH were excluded. During the 52-week treatment period, patients were randomized to receive either pitavastatin $1 \mathrm{mg}$ once daily or pitavastatin $2 \mathrm{mg}$ once daily, administered orally before breakfast. The starting dose of pitavastatin in the 2-mg group was $1 \mathrm{mg}$, and was increased to $2 \mathrm{mg}$ after Week 4. The major inclusion criteria, other than age and diagnosis, were LDL-C $\geq 190$ ( $4.9 \mathrm{mmol} / \mathrm{L})$, or $\geq 160$ $\mathrm{mg} / \mathrm{dL}(4.1 \mathrm{mmol} / \mathrm{L})$ for those who had one or more of the following risk factors: family history of coronary artery disease, obesity, type 2 diabetes mellitus, hypertension, and high-density lipoprotein cholesterol (HDL-C) $<40 \mathrm{mg} / \mathrm{dL}(1.0 \mathrm{mmol} / \mathrm{L})$. All 14 patients were randomly allocated to either treatment group (7 patients/group) and included in the safety analysis set and full analysis set (the primary population for efficacy analysis). The primary endpoint was the percentage change in the LDL-C levels from baseline to Weeks 8 and 12 . The primary analysis involved calculating the adjusted mean using a repeated measures analysis of covariance (ANCOVA) model with baseline LDL-C level as a covariate.

\section{Twelve-Week European Study ${ }^{13)}$}

A randomized, placebo-controlled, double-blind, parallel study was conducted at 10 facilities in 6 European countries to evaluate the efficacy and safety of pitavastatin in European children with high risk dyslipidemia aged 6-17 years. During the 12-week treatment period, patients were randomized to receive placebo or pitavastatin 1, 2, or $4 \mathrm{mg}$, administered orally once daily in the morning. The starting dose of pitavastatin in the 4-mg group was $2 \mathrm{mg}$, and was increased to $4 \mathrm{mg}$ after Week 4 . The major inclusion criteria other than age were LDL-C $\geq 160(4.1 \mathrm{mmol} / \mathrm{L})$, or $\geq 130 \mathrm{mg} / \mathrm{dL}$ ( $3.4 \mathrm{mmol} / \mathrm{L}$ ) for those who had one or more of the following risk factors: male, family history of premature myocardial infarction, type 2 diabetes mellitus, hypertension, HDL-C $<45 \mathrm{mg} / \mathrm{dL}(1.2 \mathrm{mmol} / \mathrm{L})$, triglycerides $(\mathrm{TG})>150 \mathrm{mg} / \mathrm{dL}(1.7 \mathrm{mmol} / \mathrm{L})$, or lipoprotein(a) $(\mathrm{Lp}(\mathrm{a}))>75 \mathrm{nmol} / \mathrm{L}$. Patients with homo- 
zygous $\mathrm{FH}$ were excluded. Genetic testing identified mutations associated with $\mathrm{FH}$ in 103 patients out of 106 randomized patients. All 106 patients randomly allocated to the study groups $(27,26,27$, and 26 patients in placebo, pitavastatin 1, 2, and $4 \mathrm{mg}$ groups, respectively) were included in the safety analysis set. Three patients who did not have lipid data after baseline were excluded, so 103 patients $(27,26,26$, and 24 patients in placebo, pitavastatin 1, 2, and $4 \mathrm{mg}$ groups, respectively) were included in the full analysis set. The primary endpoint was percentage change in LDL-C concentrations from baseline to Week 12, with imputation of missing data using the last observation carried forward (LOCF) method. The primary efficacy analysis involved calculating the adjusted mean using an ANCOVA model, with baseline LDL-C and age as covariates.

\section{Fifty-Two Week European Study ${ }^{13)}$}

To evaluate the safety and efficacy of long-term treatment with pitavastatin, a 52-week open-label extension study was conducted in 84 patients out of 103 who had completed the 12-week European study mentioned above and additional 29 patients who met the same inclusion criteria. This 52-week study was designed to enroll new patients in order to obtain sufficient exposure of pitavastatin for safety evaluation even if considerable patients who completed the first 12-week study would not agree to participate in the extension study. The starting dose of pitavastatin was $1 \mathrm{mg}$ once daily, which was increased step-wise to up to $4 \mathrm{mg}$ once daily to achieve LDL-C targets. All 112 patients who had received the test drug at least once were included in the safety analysis set and the full analysis set.

\section{Comparative Analyses for Japanese and European Studies \\ (1) Efficacy}

First, the LDL-C reduction from baseline to Week 12 in the Japanese study was analyzed applying the same method as used in the European study to the Japanese study dataset. Then, using combined data from the Japanese and European studies, factors affecting the percentage change in LDL-C from the baseline to Week 12 (LOCF) were identified among candidate factors (baseline LDL-C, age, sex, body weight, race, and country) using a stepwise method by repeatedly fitting a regression model with these factors and removing a factor shown to have a non-significant effect, with a significance level of 0.2 , on the percent change in LDL-C from the model formula. The dose level and study, and the interactions between them, were used as mandatory covariates. Identified confounding factors were then used as covariates for estimating LDL-C reduction in the combined dataset from the Japanese and European studies (1- and 2-mg dose groups), by using an ANCOVA model.

\section{(2) Safety}

The frequency and nature of adverse events were compared between the Japanese and the European studies.

\section{Results}

\section{Patient Background}

Table 1 summarizes the patient demographics in the Japanese and European studies. Although the patients in the European studies were selected to have high-risk dyslipidemia at the request of EMA, all of them had primary dyslipidemia and almost all had heterozygous $\mathrm{FH}$, confirmed by genetic testing in the 12-week study (103 of 106 (97.2\%) patients) and in the 52- week study (107 of $112(95.5 \%))$. The mean age of the subjects was higher in the Japanese study compared to the European study (11.8 and 10.6 years, respectively). The mean body weight, height, and body mass index (BMI) were all lower in the Japanese study compared to the European study $(41.1$ and $43.6 \mathrm{~kg}$, 147.6 and $148.2 \mathrm{~cm}$, and 18.7 and $19.1 \mathrm{~kg} / \mathrm{m}^{2}$, respectively). All the subjects in the Japanese study were boys while more than half $(54.7 \%)$ of those in the European study were girls.

\section{Efficacy}

Table 2 shows the percentage change in LDL-C from baseline to Week 12 in the Japanese and European studies when analyzed using the same method. LDL-C levels were reduced by 28.5 and $36.3 \%$ in the 1 - and 2-mg groups, respectively in the Japanese study, which was numerically higher than in the European study (23.5 and $30.1 \%$, respectively. Then, using the combined dataset, the above-mentioned stepwise method identified baseline LDL-C, age, and body weight as factors affecting the percentage LDL-C change based on the pre-specified criterion of $p<0.2$ (Table 3). Including these identified factors into the model, the LDL-C reductions with pitavastatin 1 and $2 \mathrm{mg}$ from the two studies were adjusted for these factors. As a result, there was no significant difference in the percentage LDL-C reduction in the Japanese study (24.5 and $33.5 \%$ in the $1-$ and $2-\mathrm{mg}$ groups, respectively) compared with the European study (23.6 and 30.8\% in the 1- and 2-mg groups, respectively) (Table 4).

\section{Safety}

The Japanese study remained double-blind through its 52-week treatment period. The 12-week European 
Table 1. Patient demographics

\begin{tabular}{|c|c|c|c|c|c|c|c|c|}
\hline & \multicolumn{3}{|c|}{ Japanese study } & \multicolumn{5}{|c|}{ European study } \\
\hline & $\begin{array}{c}\text { Pitavastatin } \\
1 \mathrm{mg}\end{array}$ & $\begin{array}{l}\text { Pitavastatin } \\
\quad 2 \mathrm{mg}\end{array}$ & Total & Placebo & $\begin{array}{c}\text { Pitavastatin } \\
1 \mathrm{mg}\end{array}$ & $\begin{array}{l}\text { Pitavastatin } \\
2 \mathrm{mg}\end{array}$ & $\begin{array}{l}\text { Pitavastatin } \\
\quad 4 \mathrm{mg}\end{array}$ & Total \\
\hline $\mathrm{N}$ & 7 & 7 & 14 & 27 & 26 & 27 & 26 & 106 \\
\hline Age, years & $12.0(1.4)$ & $11.6(1.8)$ & $11.8(1.6)$ & $10.4(3.3)$ & $10.5(2.8)$ & $11.1(2.9)$ & $10.3(2.7)$ & $10.6(2.9)$ \\
\hline Male & $7(100)$ & $7(100)$ & $14(100)$ & $12(44.4)$ & $12(46.2)$ & $10(37.0)$ & $14(53.8)$ & $48(45.3)$ \\
\hline \multicolumn{9}{|l|}{ Race } \\
\hline White/Caucasian & 0 & 0 & 0 & $26(96.3)$ & $24(92.3)$ & $26(96.3)$ & $26(100.0)$ & $102(96.2)$ \\
\hline Asian & $7(100)$ & $7(100)$ & $14(100)$ & $1(3.7)$ & $1(3.8)$ & 0 & 0 & $2(1.9)$ \\
\hline Black/African or African American & 0 & 0 & 0 & 0 & 0 & $1(3.7)$ & 0 & $1(0.9)$ \\
\hline Multiple races & 0 & 0 & 0 & 0 & $1(3.8)$ & 0 & 0 & $1(0.9)$ \\
\hline Height, $\mathrm{cm}$ & $145.1(12.6)$ & $150.0(13.1)$ & $147.6(12.6)$ & $145.7(16.9)$ & $149.7(18.0)$ & $152.0(15.2)$ & $145.6(15.7)$ & $148.2(16.5)$ \\
\hline Weight, kg & $40.4(11.2)$ & $41.7(9.6)$ & $41.1(10.1)$ & $40.5(16.4)$ & 46.5 (20.9) & $47.6(16.6)$ & $39.5(12.1)$ & $43.6(16.9)$ \\
\hline BMI, $\mathrm{kg} / \mathrm{m}^{2}$ & $19.0(4.4)$ & $18.3(2.2)$ & $18.7(3.3)$ & $18.3(3.5)$ & $19.7(4.9)$ & $20.0(3.8)$ & $18.2(2.8)$ & $19.1(3.9)$ \\
\hline
\end{tabular}

Data are presented as mean (SD) for continuous parameters or number of patients (\%) for categorical ones.

BMI, body mass index.

Table 2. Baseline and percentage change from baseline to Week 12 in LDL-C adjusted for baseline LDL-C and age separately estimated in each study dataset

\begin{tabular}{lccccccc}
\hline & \multicolumn{2}{c}{ Japanese study } & & \multicolumn{4}{c}{ European study } \\
\cline { 2 - 3 } & Pitavastatin & Pitavastatin & & Pitavastatin & Pitavastatin & Pitavastatin & Placebo \\
\hline $\mathrm{N}$ & $1 \mathrm{mg}$ & $2 \mathrm{mg}$ & & $1 \mathrm{mg}$ & $2 \mathrm{mg}$ & $4 \mathrm{mg}$ & 27 \\
Baseline, $\mathrm{mg} / \mathrm{dL}$ & 7 & 7 & & 26 & 26 & 24 & 27 \\
Adjusted percentage & $245.4(68.1)$ & $269.6(51.2)$ & & $231.4(45.5)$ & $223.1(35.9)$ & $240.7(54.3)$ & $240.5(69.0)$ \\
change & -28.5 & -36.3 & & -23.5 & -30.1 & -39.3 & 1.0 \\
\hline
\end{tabular}

Data are presented as mean (SD) for baseline values and least squares mean (95\% CI) for adjusted percentage change estimated using ANCOVA with baseline level and age as covariates.

ANCOVA, analysis of covariance; SD, standard deviation; CI, confidence interval.

study was double-blind, followed by a 52-week openlabel study, and additional patients were enrolled in the longer term follow up study. Pitavastatin was well tolerated without any difference in the frequency and nature of adverse events between the treatment groups or between the studies in Japan and Europe. During the double-blind period, one patient in each region had a serious adverse event; however, neither event was considered to be related to pitavastatin treatment. Two patients discontinued medication from the European study due to adverse events while no discontinuation was seen in the Japanese study. In the Japanese study, there were no adverse events considered causally related to pitavastatin, but in the European study 16 (15.1\%) of the 106 subjects reported events, including abdominal pain in $5(4.7 \%)$, headache in $3(2.8 \%)$, and abdominal discomfort and vomiting in $2(1.9 \%)$ each, that were considered causally related. In the
European study, the overall frequency of adverse events, the frequency of events considered to be causally related to pitavastatin, and the nature of the events reported were well balanced across the treatment groups including placebo. All but one of the adverse events were mild. One patient $(0.9 \%)$ in the $4 \mathrm{mg}$ group had moderate myalgia. In the Japanese study, the most frequent adverse events were seasonal allergy, gastroenteritis, nasopharyngitis, pharyngitis, ligament sprain, nasal bleeding, allergic rhinitis, upper airway inflammation, and eruption. Similarly, the most frequent adverse events in the European study were nasopharyngitis, influenza, viral upper airway infection, abdominal pain, abdominal discomfort, vomiting, and headache. During the European open-label 52-week study, pitavastatin $4 \mathrm{mg}$ was administered to 103 patients, and the frequency of adverse events considered to be causally related to pitavastatin was $6.8 \%$ (7 patients). There- 
Table 3. Solution for fixed effects as a result of stepwise method to identify factors affecting the percentage change in LDL-C from the combined dataset of Japanese and European study

\begin{tabular}{llcccc}
\hline \multicolumn{1}{c}{ Effect } & Study Identifier & Dose & Estimate & SEM & $p$ value \\
\hline Intercept & & -25.1534 & 12.1494 & 0.0428 \\
Dose & & 9.0138 & 5.8351 & 0.1278 \\
Dose & & 0 & - & - \\
Baseline LDL-C & & -0.04628 & 0.03183 & 0.1513 \\
Age & & -1.0615 & 0.8811 & 0.2331 \\
Body Weight & & 0.3086 & 0.1294 & 0.0203 \\
Study Identifier & European study & & 2.7080 & 4.9292 & 0.5848 \\
Study Identifier & Japanese study & & 0 & - & - \\
Dose* Study Identifier & European study & $1 \mathrm{mg}$ & -1.8201 & 6.6207 & 0.7843 \\
Dose* Study Identifier & Japanese study & $1 \mathrm{mg}$ & 0 & - & - \\
Dose* Study Identifier & European study & $2 \mathrm{mg}$ & 0 & - & - \\
Dose* Study Identifier & Japanese study & $2 \mathrm{mg}$ & 0 & - & - \\
\hline
\end{tabular}

SEM, standard error of mean

Table 4. Percentage change from baseline to Week 12 in LDL-C adjusted for identified factors as covariates in pooled dataset of Japanese and European study

\begin{tabular}{|c|c|c|c|c|}
\hline & \multicolumn{2}{|c|}{ Japanese study } & \multicolumn{2}{|c|}{ European study } \\
\hline Adjusted percentage change & $-24.5(-33.2,-15.8)$ & $-33.5(-42.2,-24.8)$ & $-23.6(-28.0,-19.2)$ & $-30.8(-35.1,-26.5)$ \\
\hline$p$ & - & - & 0.861 & 0.585 \\
\hline
\end{tabular}

Data are presented as least squares mean (95\% CI) estimated using ANCOVA with baseline level, age, and body weight as covariates.

ANCOVA, analysis of covariance; SD, standard deviation; CI, confidence interval.

fore, the rate, severity, and nature of adverse events throughout the 52-week follow-up were similar to that during the 12-week double-blind period. There was no significant change in sex hormone levels in either study.

\section{Discussion}

In both the Japanese and European studies, conducted in compliance with GCP guidelines, pitavastatin resulted in LDL-C lowering in a similar dosedependent manner in children and adolescents with $\mathrm{FH}$. The LDL-C lowering efficacy in the Japanese study was numerically larger by $5 \%$ to $6 \%$ than that in the European study when the studies were analyzed using the same method. However, baseline characteristics differed between the two studies, and these factors were found to affect the change in LDL-C concentration during the study. In particular baseline LDL-C, age, and body weight were shown to have an effect on the LDL-C lowering capacity of pitavastatin. When these factors were taken into account, the dif- ference in LDL-C reduction between the studies became insignificant. This suggests that the LDL-C lowering efficacy of pitavastatin in the Japanese and European children and adolescents was similar if their baseline LDL-C, age, and body weight were similar, regardless of other intrinsic ethnic factors. Especially for the higher baseline LDL-C in the Japanese study, that might be attributable to the higher upper limit $(30$ $\mathrm{mg} / \mathrm{dL}$ higher) of LDL-C for inclusion criterion in the Japanese study. Concomitant use of lipid-lowering drugs other than pitavastatin was discouraged in both studies, so extrinsic factors were also unlikely to have had a major interaction with the efficacy of pitavastatin. Our finding is in line with the observed effect of pitavastatin in adult Caucasian and Japanese patients, where it was shown that LDL-C lowering efficacy of pitavastatin was similar in the studies in patients with $\mathrm{FH}$ or other forms of hypercholesterolemia ${ }^{14,15)}$ and in the bridging analysis of the data in Japan, Europe and the United States performed for the supplemental application submitted in Japan (unpublished). Pharmacokinetic profiles of pitavastatin were also similar 
between healthy Caucasian and Japanese men after adjusting for age and body weight ${ }^{16)}$. The present finding is reassuring, as it shows that the efficacy profile of pitavastatin is not influenced by ethnic background other than baseline LDL-C, age, and body weight. It has been suggested that body weight, age and baseline level of LDL-C have an effect on the LDL-C lowering effect of statins and this effect was also observed in our study. However, pitavastatin is the first statin to be studied in children and adolescents with $\mathrm{FH}$ in both Japan and Europe to allow a comparison of ethnicity on efficacy. The baseline parameters were found not to have an effect on the safety profile, and as such, pitavastatin can safely be prescribed, irrespective of the age and body composition of the patient. It is of note that the full range of dosing, as approved for adults, was investigated in the studies conducted in Europe and the approved doses of pitavastatin in adults are the same in Japan, Europe and the United States while those of other statins in Japan are half of those in Europe and the United States. Therefore, in daily clinical practice for the treatment of similar populations to those covered in these clinical trials, no adjustment of dose based on age is necessary when pitavastatin is used in children and adolescents.

Most of the major treatment guidelines for $\mathrm{FH}$ recently published in Western countries do not limit the use of statins to post-pubertal children as was formerly the case ${ }^{2,6,9,10,17-19)}$. A Cochrane Review, which systematically evaluated multiple clinical studies in children with $\mathrm{FH}$, did not find any influence of statin therapy on sexual maturation ${ }^{20)}$, and the 10-year follow-up data on the efficacy and safety of statins in children with $\mathrm{FH}$ also did not show an effect of statins on length/growth ${ }^{21}$. Furthermore, no evidence of a significant reduction in sex hormones was found in these studies with pitavastatin in children and adolescents, suggesting that pitavastatin likely has no effect on sexual maturation. No girls were enrolled in the Japanese study; however, both boys and girls aged 6-17 years were enrolled in the European study which showed no significant difference in efficacy and safety between the sexes. Therefore, we may expect similar efficacy and safety of pitavastatin in Japanese girls with $\mathrm{FH}$ as well. Nevertheless, we are awaiting the results of an ongoing post-marketing surveillance study for the safety and efficacy of this drug in Japanese children and adolescents, including girls.

This study has a number of limitations. First, the sample size and treatment duration of the studies were insufficient to draw robust conclusions about long term safety and efficacy. The limited number of patients in the Japanese study, i.e. 14, makes it difficult to exclude the possibility that the present results were drawn by chance. Besides, these Japanese patients were not identified based on genetic testing and the Japanese study did not enroll girls with FH. Although the results of the European study suggest there are similarities in the efficacy and safety between the sexes, no data on the use of pitavastatin in Japanese girls is currently available. There is no clinical trial evidence in the treatment of children younger than 6 years. There is a case report of pitavastatin treatment for 6 months in 4-year-old dichorionic diamniotic twins (boy and girl) with $\mathrm{FH}^{22}$. Further studies are awaited to accumulate evidence in greater numbers of patients, longer-term treatment, and treatment of an even younger population. Current international collaborative efforts may promote early detection and treatment of $\mathrm{FH}$ and result in the accumulation of such evidence ${ }^{23,24)}$.

\section{Conclusion}

In this comparative study we observed no significant differences in the safety and efficacy profile of pitavastatin in Japanese and European children and adolescents with FH. This suggests that ethnicity has, at most, a minor effect on the clinical outcome of pitavastatin treatment in those patients. The data currently available on the efficacy and safety of pitavastatin were derived from a limited number of patients in studies with a maximum duration of treatment of 15 months.

The present analysis has provided useful information for the management of children and adolescents with FH in Japan and Europe. Although data on pitavastatin use in Japanese girls with $\mathrm{FH}$ are unavailable, the analysis revealed no information that raises special concerns related to sex difference. These findings might be beneficial in the clinical use of pitavastatin for the treatment of children and adolescents with $\mathrm{FH}$ in Japan, and in Europe once the indication is approved.

\section{Acknowledgments}

The authors express sincere appreciation to the doctors who provided advice on this study. Dr. Neil Hounslow, Kowa Research Europe. Ltd., contributed to edit the manuscript through meticulously coordinating authors' comments. The original studies and this pooled analysis were carried out by Kowa Company, Ltd., Tokyo, Japan.

\section{Conflicts of Interest}

M.H.-S. reports personal fees from Kowa, during the conduct of the study, grants from Astellas, Kaneka 
Medics, MSD, Takeda, personal fees from Astellas Amgen, Astellas, Sanofi, Astrazeneca, JCR Pharm, Daiichi-Sankyo, MSD, Phizer, Torii, Shionogi, outside the submitted work. J.J.P.K. reports personal fees from Kowa, during the conduct of the study, personal fees from Affiris, Akarna, Amgen, CSL, Behring, Staten Biotech, Regeneron, Madrigal, The Medicines Company, Esperion, Gemphire, Akcea, Ionis, outside the submitted work. G.K.H. reports grants from Netherlands Organisation for Scientific Research, grants from Cardiovascular Research Initiative, grants from European Union, personal fees and non-financial support from AMGEN, personal fees and non-financial support from Aegerion, personal fees from Pfizer, personal fees and non-financial support from AstraZeneca, personal fees and non-financial support from Sanofi, personal fees from Regeneron, personal fees from Kowa, personal fees from IONIS, personal fees from Cerenis, non-financial support from Synageva, outside the submitted work. K.K.R. reports grants from Sanofi, Regeneron, Pfizer, Amgen, MSD, personal fees from Sanofi, Amgen, Regeneron, Lilly, Medicines Company, AstraZeneca, Pfizer, Kowa, Algorithm, IONIS, Esperion, Novo Nordisk, Takeda, Boehringer Ingelheim, Resverlogix, Abbvie, outside the submitted work. A.O. has nothing to disclose. O.A. has nothing to disclose. T.O. has nothing to disclose. T.O. has nothing to disclose. H.S. is an employee of Kowa. A.W. has nothing to disclose.

\section{References}

1) Harada-Shiba $M$, Arai H, Oikawa $S$, Ohta T, Okada T, Okamura T, Nohara A, Bujo H, Yokote K, Wakatsuki A, Ishibashi $S$ and Yamashita S: Guidelines for the management of familial hypercholesterolemia. J Atheroscler Thromb, 2012; 19: 1043-1060

2) Nordestgaard BG, Chapman MJ, Humphries SE, Ginsberg HN, Masana L, Descamps OS, Wiklund O, Hegele RA, Raal FJ, Defesche JC, Wiegman A, Santos RD, Watts GF, Parhofer KG, Hovingh GK, Kovanen PT, Boileau C, Averna M, Boren J, Bruckert E, Catapano AL, Kuivenhoven JA, Pajukanta P, Ray K, Stalenhoef AF, Stroes E, Taskinen MR and Tybjaerg-Hansen A: Familial hypercholesterolaemia is underdiagnosed and undertreated in the general population: guidance for clinicians to prevent coronary heart disease: consensus statement of the European Atherosclerosis Society. Eur Heart J, 2013; 34: 34783490a

3) Mabuchi H, Nohara A, Noguchi T, Kobayashi J, Kawashiri MA, Tada H, Nakanishi C, Mori M, Yamagishi M, Inazu A and Koizumi J: Molecular genetic epidemiology of homozygous familial hypercholesterolemia in the Hokuriku district of Japan. Atherosclerosis, 2011; 214: 404-407

4) Zhou M and Zhao D: Familial Hypercholesterolemia in
Asian Populations. J Atheroscler Thromb, 2016; 23: 539549

5) Harada-Shiba M, Arai H, Okamura T, Yokote K, Oikawa S, Nohara A, Okada T, Ohta T, Bujo H, Watanabe M, Wakatsuki A and Yamashita S: Multicenter study to determine the diagnosis criteria of heterozygous familial hypercholesterolemia in Japan. J Atheroscler Thromb, 2012; 19: 1019-1026

6) Wiegman A, Gidding SS, Watts GF, Chapman MJ, Ginsberg HN, Cuchel M, Ose L, Averna M, Boileau C, Boren J, Bruckert E, Catapano AL, Defesche JC, Descamps OS, Hegele RA, Hovingh GK, Humphries SE, Kovanen PT, Kuivenhoven JA, Masana L, Nordestgaard BG, Pajukanta P, Parhofer KG, Raal FJ, Ray KK, Santos RD, Stalenhoef AF, Steinhagen-Thiessen E, Stroes ES, Taskinen MR, Tybjaerg-Hansen A and Wiklund O: Familial hypercholesterolaemia in children and adolescents: gaining decades of life by optimizing detection and treatment. Eur Heart J, 2015; 36: 2425-2437

7) Verschuren WM, Jacobs DR, Bloemberg BP, Kromhout D, Menotti A, Aravanis C, Blackburn H, Buzina R, Dontas AS, Fidanza F, Karvonen MJ, Nedelijković S, Nissinen $A$ and Toshima H: Serum total cholesterol and long-term coronary heart disease mortality in different cultures. Twentyfive-year follow-up of the seven countries study. JAMA, 1995; 274: 131-136

8) Saito I, Folsom AR, Aono H, Ozawa H, Ikebe $T$ and Yamashita T: Comparison of fatal coronary heart disease occurrence based on population surveys in Japan and the USA. Int J Epidemiol, 2000; 29: 837-844

9) Watts GF, Gidding S, Wierzbicki AS, Toth PP, Alonso R, Brown WV, Bruckert E, Defesche J, Lin KK, Livingston M, Mata P, Parhofer KG, Raal FJ, Santos RD, Sijbrands EJ, Simpson WG, Sullivan DR, Susekov AV, Tomlinson B, Wiegman A, Yamashita S and Kastelein JJ: Integrated guidance on the care of familial hypercholesterolaemia from the International $\mathrm{FH}$ Foundation. Int J Cardiol, 2014; 171: 309-325

10) Expert Panel on Integrated Guidelines for Cardiovascular Health and Risk Reduction in Children and Adolescents; National Heart L, and Blood Institute.: Expert panel on integrated guidelines for cardiovascular health and risk reduction in children and adolescents: summary report. Pediatrics, 2011; 128 Suppl 5: S213-256

11) Harada-Shiba M, Ohta T, Ohtake A, Dobashi K, Nohara A, Yamashita S and Yokote K: Japan Pediatric Society and Japan Atherosclerosis Society (eds.). [2017 practice guide for the treatment of pediatric familial hypercholesterolemia in Japan], Article in Japanese, Japan Atherosclerosis Society, 2017

12) Harada-Shiba M, Arisaka O, Ohtake A, Okada $T$ and Suganami H: Efficacy and Safety of Pitavastatin in Japanese Male Children with Familial Hypercholesterolemia. J Atheroscler Thromb, 2016; 23: 48-55

13) Braamskamp MJ, Stefanutti C, Langslet G, Drogari E, Wiegman A, Hounslow N and Kastelein JJ: Efficacy and Safety of Pitavastatin in Children and Adolescents at High Future Cardiovascular Risk. J Pediatr, 2015; 167: 338343. e5

14) Saito $Y$, Yamada N, Teramoto T, Itakura H, Hata $Y$, Nakaya N, Mabuchi H, Tushima M, Sasaki J, Goto Y and 
Ogawa N: Clinical efficacy of pitavastatin, a new 3-hydroxy-3-methylglutaryl coenzyme A reductase inhibitor, in patients with hyperlipidemia. Dose-finding study using the double-blind, three-group parallel comparison. Arzneimittelforschung, 2002; 52: 251-255

15) Stender S, Budinski D, Gosho $M$ and Hounslow N: Pitavastatin shows greater lipid-lowering efficacy over 12 weeks than pravastatin in elderly patients with primary hypercholesterolaemia or combined (mixed) dyslipidaemia. Eur J Prev Cardiol, 2013; 20: 40-53

16) Warrington $S$, Nagakawa $S$ and Hounslow N: Comparison of the pharmacokinetics of pitavastatin by formulation and ethnic group: an open-label, single-dose, twoway crossover pharmacokinetic study in healthy Caucasian and Japanese men. Clin Drug Investig, 2011; 31: 735-743

17) National Cholesterol Education Program (NCEP): highlights of the report of the Expert Panel on Blood Cholesterol Levels in Children and Adolescents. Pediatrics, 1992; 89: 495-501

18) Daniels SR and Greer FR: Lipid screening and cardiovascular health in childhood. Pediatrics, 2008; 122: 198-208

19) Daniels SR, Gidding SS and de Ferranti SD: Pediatric aspects of familial hypercholesterolemias: recommendations from the National Lipid Association Expert Panel on Familial Hypercholesterolemia. J Clin Lipidol, 2011; 5: S30-37

20) Vuorio A, Kuoppala J, Kovanen PT, Humphries SE, Tonstad S, Wiegman A and Drogari E: Statins for children with familial hypercholesterolemia. Cochrane Database Syst Rev, 2014; 7: CD006401

21) Kusters DM, Avis HJ, de Groot E, Wijburg FA, Kastelein JJ, Wiegman A and Hutten BA: Ten-year follow-up after initiation of statin therapy in children with familial hypercholesterolemia. JAMA, 2014; 312: 1055-1057

22) Miyagi Y, Harada-Shiba M and Ohta T: Effect of Statin Therapy in 4-Year-Old Dichorionic Diamniotic Twins with Familial Hypercholesterolemia Showing Multiple Xanthomas. J Atheroscler Thromb, 2016; 23: 112-117

23) Pang J, Lansberg PJ and Watts GF: International Developments in the Care of Familial Hypercholesterolemia: Where Now and Where to Next? J Atheroscler Thromb, 2016; 23: 505-519

24) Watts GF, Ding PY, George P, Hagger MS, Hu M, Lin J, Khoo KL, Marais AD, Miida T, Nawawi HM, Pang J, Park JE, Gonzalez-Santos LB, Su TC, Truong TH, Santos RD, Soran H, Yamashita S and Tomlinson B: Translational Research for Improving the Care of Familial Hypercholesterolemia: The "Ten Countries Study" and Beyond. J Atheroscler Thromb, 2016; 23: 891-900 